\title{
Forward and backward second-order Pavlovian conditioning in honeybees
}

\author{
Syed Abid Hussaini, ${ }^{1,3}$ Bernhard Komischke, ${ }^{1,3}$ Randolf Menzel, ${ }^{1,4,5}$ \\ and Harald Lachnit ${ }^{2}$ \\ ${ }^{1}$ Neurobiology, Institute of Biology, Free University of Berlin, D-14195 Berlin, Germany; ${ }^{2}$ Faculty of Psychology,
Philipps-Universität Marburg, D-35032 Marburg, Germany
}

Second-order conditioning (SOC) is the association of a neutral stimulus with another stimulus that had previously been combined with an unconditioned stimulus (US). We used classical conditioning of the proboscis extension response (PER) in honeybees (Apis mellifera) with odors (CS) and sugar (US). Previous SOC experiments in bees were inconclusive, and, therefore, we attempted to demonstrate SOC in the following three experiments: (Experiment 1) After differential conditioning (pairing odor A with US and presenting odor B without US), the bees experienced two pairs of partially overlapping odors, either a new odor $C$ followed by a previously reinforced odor $A(C-A)$ or a new odor $C$ followed by a previously nonreinforced odor B (C-B). (Experiment 2) After differential conditioning, bees were presented with C-A or A-C. (Experiment 3) Bees were first presented with C-A or A-C before differential conditioning and were tested with odor C. We observed: (Experiment 1) $40 \%$ of the bees showed PER to the C-A presentation, but only $20 \%$ showed PER to the C-B presentation. (Experiment 2) $40 \%$ of the bees showed PER to the C-A presentation, while only $20 \%$ showed PER to the reversed sequence A-C. Experiments 1 and 2 showed that a previously reinforced odor can be a secondary reinforcer for excitatory SOC only with forward-pairing. (Experiment 3) PER toward $C$ was lower (15\%) in bees presented with A-C than with C-A $(25 \%)$. This showed that backward SOC is not as effective as forward SOC. These results help to delineate different conditions that are critical for the phenomenon of SOC.

\begin{abstract}
Associative learning enables organisms to internally represent correlations among environmental events. Animals and humans associate one cue with another when one of them is biologically relevant. This is the simplest form of association, called firstorder conditioning. In addition, associative learning can be indirect or of second-order, as in the case of Pavlov's dog (Pavlov 1927). A dog, when put into a dark room and given food (primary reinforcer, US) just before switching on the light (conditioned stimulus, CS), salivated (conditioned response, CR) every time the light was switched on (CS leads to CR). Then, in a separate event, the dog was presented with the tone of a bell (neutral stimulus, NS) followed by the light (CS, a secondary reinforcer). After several such combinations, the dog already salivated in the presence of the tone, although it never had been combined with the primary reinforcer food. Pavlov called the latter process "reflex of the second order," which is now known as second-order conditioning (SOC).

In nature, animals have to relate one stimulus with another consistently and precisely in order to acquire food, find mates, and escape predators. Insects such as honeybees have to come across certain stimuli that have no direct meaning. They have to decipher random stimuli such as trees, buildings, flowers, odors, etc. into meaningful information. For example, during foraging trips honeybees encounter several odors, and not all odors lead to a food source. But certainly some odors might serve as a clue to reach another odor that might be the source of food. Making secondary associations such as these toward biologically relevant
\end{abstract}

${ }^{3}$ These authors contributed equally to this work.

4Present address: AG Neurobiologie, Königin-Luise-Strasse 28/30, 14195 Berlin, Germany.

${ }^{5}$ Corresponding author.

E-mail menzel@neurobiologie.fu-berlin.de; fax 49-30-83855455.

Article is online at http://www.learnmem.org/cgi/doi/10.1101//m.471307. stimuli is a complex learning task requiring complex information processing of the brain.

The phenomenon of SOC has been studied in many animal species, e.g., monkeys (Findley et al. 1966), rats (Kamil 1969; Holland and Rescorla 1975), pigeons (Rescorla 1979; Stanhope 1992), zebrafish (Hall and Suboski 1995), and goldfish (Amiro and Bitterman 1980). It was also observed in invertebrates like mollusks (Sahley 1981), Aplysia (Hawkins et al. 1998), fruit flies (Brembs and Heisenberg 2001), and honeybees (Takeda 1961; Bitterman et al. 1983; Menzel 1990).

The experiments by Takeda (1961) and Bitterman et al. (1983) had already shown some evidence of SOC but were inconclusive due to insufficient controls (like pairing effect and backward SOC control) and a small number of animals $(<10)$. The present three experiments (for overview, see Table 1) not only attempt to overcome these insufficiencies but also to delineate the conditions being critical for the occurrence of SOC. The exact sequence of stimuli that triggers SOC is not known and also which different learning processes are involved during SOC or reversal of SOC phases is not known. To understand this, starting from Experiment 1 we manipulated different parameters such as sequence of stimuli (Experiment 2) and the protocol sequence (Experiment 3).

We used odors as CSs and sugar reinforcement as US, to train the honeybees in an appetitive Pavlovian conditioning paradigm called the proboscis extension response (PER) (Kuwabara 1957; Bitterman et al. 1983). Our experiments show that bees learn second-order associations only under certain special conditions.

\section{Materials and Methods}

Foraging honeybees (Apis mellifera) were caught in a transparent box at the entrance of the outdoor hive $2 \mathrm{~h}$ prior to training and were cold-anesthetized on ice. The immobile bees were harnessed 
Table 1. Experimental design

\begin{tabular}{llccc}
\hline $\begin{array}{l}\text { Experiment } \\
\text { no. }\end{array}$ & Group type & Phase 1 & Phase 2 & Test \\
\hline Experiment 1 & Group Plus & A-US; B-noUS & C-A & - \\
& Group Minus & A-US; B-noUS & C-B & - \\
Experiment 2 & Group Forward & A-US; B-noUS & C-A & - \\
& Group Backward & A-US; B-noUS & A-C & - \\
Experiment 3 & Group Forward & C-A & A-US; B-noUS & C \\
& Group Backward & A-C & A-US; B-noUS & C \\
\hline
\end{tabular}

in metal tubes such that only the mandibles, proboscis, and antennae could move freely (Bitterman et al. 1983). Ten minutes before training, bees were checked for PER (unconditioned response, UR) by lightly touching the antennae with a 30\% sucrose solution (US). Only bees that demonstrated the UR were trained ( $<5 \%$ were discarded). Citral, geraniol, 2-hexanal, nonanone, 2-octanol, and limonene were used as odor CSs, referred to as A, $\mathrm{B}$, and $\mathrm{C}$ in later sections. A computer-driven odor device, blowing a continuous stream of air over the antennae, was used to deliver the odors to the bees (Galizia et al. 1997; Komischke et al. 2002). Four $1-\mathrm{mL}$ syringes, each containing $4 \mu \mathrm{L}$ of the desired odor on a half square inch of filter paper, were fitted into the holes of the odor-delivering device (Galizia et al. 1997). Each bee was fixed in front of this device with its antennae facing the air stream, and an exhaust system behind the bee removed the odor.

\section{Experimental procedures}

The bees were kept within the air stream of the odor-delivering device for a total of $60 \mathrm{sec}$ during each trial; $26 \mathrm{sec}$ before and 28 sec after the stimulus and 6 sec during the presentation of the test stimuli. During the 6-sec stimulus time, stimulus- 1 and stimulus- 2 were presented for $4 \mathrm{sec}$ and $3 \mathrm{sec}$, respectively, with a $1 \mathrm{sec}$ overlap between the two. In all phases of all experiments, we used five trials per stimulus and the inter-trial interval (ITI) was always $12 \mathrm{~min}$. The differential conditioning phase started with a presentation of odor A for $4 \mathrm{sec}$ that was subsequently reinforced with $30 \%$ sucrose solution (US) for $3 \mathrm{sec}$, with an overlap of $1 \mathrm{sec}$ between A and US. In the next trial, a second odor B was presented but without any US reinforcement. The percent proboscis extension response (\% PER) of the bees as observed during the first $2 \mathrm{sec}$ of odor presentation was calculated for A and B.

In the SOC phase, two odors were used. Throughout this phase, the bees were never reinforced with US. The first odor was presented for $4 \mathrm{sec}$, followed by the second odor for $3 \mathrm{sec}$. The overlap between these two odors was $1 \mathrm{sec}$. The \% PER to the first odor was recorded beginning at onset for $2 \mathrm{sec}$. Thus the recording ended before onset of the second odor. In addition, in Experiments 2 and 3 responses to the second odor were recorded for $2 \mathrm{sec}$ after the offset of odor 1. A computer beep signaled the timing for the presentation. Furthermore, onset and offset of an odor was indicated by audible clicks from the solenoid valves (a part of the odor device).

Each of the three experiments consisted of two groups and at least two phases. The different phases followed each other immediately. All groups in all experiments experienced the same differential conditioning protocol (A-US; B-noUS). Experiments 1 and 2 studied SOC in the conventional manner, i.e., differential conditioning in Phase 1 was followed by an SOC protocol in Phase 2. This we call "forward SOC." In Experiment 1, a new odor $\mathrm{C}$ was followed by odor A that was initially paired with the US in "Group Plus" (C-A), while in "Group Minus," C was followed by $\mathrm{B}$, the odor that was initially presented without the US (C-B). Successful SOC can be inferred from a between-group comparison of response to $\mathrm{C}$ at the end of Phase 2. "Group Forward" of Ex- periment 2 experienced exactly the same sequence of differential conditioning and SOC as "Group Plus" of Experiment 1 (forward SOC with forward-pairing C-A). "Group Backward" of Experiment 2, however, received A-C pairings instead of C-A pairings in Phase 2 after the very same differential conditioning protocol as the other three groups (forward SOC with backward-pairing A-C). Hence, in Experiment 2 the sequence of stimuli was reversed (forward-pairing versus backward-pairing). In Experiment 2, successful SOC can be evaluated by comparing response to the first and last presentations of $\mathrm{C}$ in Phase 2 within-groups and also by between-group comparison of the fourth and fifth trials of each group.

Finally, Experiment 3 is the same as Experiment 2, but the Phase 1 and Phase 2 protocols were reversed ("backward SOC"). In this experiment, differential conditioning took place in Phase 2, after C-A pairings (Group Forward: forward-pairing) or A-C pairings (Group Backward: backward-pairing) were administered in Phase 1. This backward SOC can also be looked at as two variants of sequential sensory preconditioning (see Müller et al. 2000). In order to evaluate SOC, response to $C$ at the first test trial in Phase 3 was compared to response to $\mathrm{C}$ in the last trial of Phase 1 within-groups.

\section{Dependent variable and statistics}

The PER is a clear response that leads to either a full extension of the proboscis or no response. Therefore, publications based on PER scoring involve video recording only when quantitative measures need to be taken (e.g., latency of PER, duration of PER). This was not the case in our study. There was another reason why it was also not necessary to run blind experiments. The experiments involved a large number of animals that were marked beforehand by the experimenter with numbers. During this procedure, the different experimental groups were shuffled in such a way that sequential animals belonging to the different groups were treated at the same intervals and spaced out equally throughout the experimental period. It was, therefore, impossible for the experimenter to keep track of animals belonging to the same experimental group.

Between-group comparisons were analyzed using the Kruskal-Wallis ANOVA and $\chi^{2}$-tests. Within-group comparisons were performed with the Wilcoxon matched-pairs test and the McNemar test. The alpha-level was set to 0.05 for all analyses.

\section{Results}

Experiment 1: Does olfactory SOC occur in honeybees?

The odors used as A, B, and C were citral, geraniol, or 2-hexanal. They were equally balanced and used in all possible combinations to exclude odor-specific effects. The first column of Figure 1 shows that the acquisition curves for the differential conditioning protocol in Phase 1 were quite similar for both groups $(n=60$ each). The middle column of Figure 1 shows response to the new odor C for forward-pairing with A (C-A) in Group Plus (upper quarter) and for forward-pairing with B (C-B) in Group Minus (lower quarter). Comparisons between groups by Kruskal-Wallis ANOVA showed significantly higher response for forwardpairing with A compared to forward-pairing with $\mathrm{B}$ in Trial 4 $(H=4.56, P<0.05, \mathrm{df}=1)$ and in Trial $5(H=7.01, P<0.01$, $\mathrm{df}=1)$.

In both groups, the \% PER to the stimuli was recorded in three parts: first stimulus alone, overlap, second stimulus alone. Therefore we were able to take a look at the extinction of $\mathrm{A}$ in Group Plus and B in Group Minus (see Fig. 1, third column). Responding to A (the secondary reinforcer) did not decrease from Trial 1 to Trial $5\left(\chi^{2}=0.75, \mathrm{~ns}, \mathrm{df}=1\right)$, nor did response to $\mathrm{B}$ 


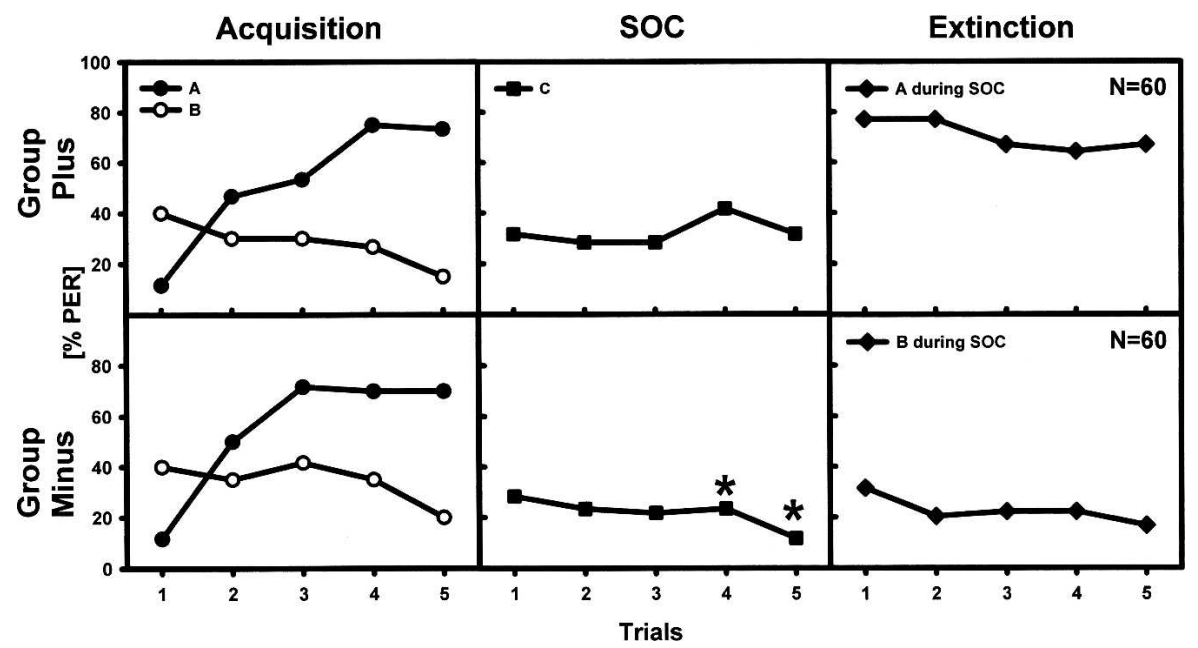

Figure 1. Second-order conditioning (SOC) in honeybees. Acquisition of odors A and B (left panel) in a differential conditioning design (A-US, B-noUS). During the SOC phase (right panel), Group Plus received pairings of a new odor $\mathrm{C}$ with an already-conditioned excitatory odor $\mathrm{A}$, while Group Minus received pairings of a new odor $\mathrm{C}$ with an odor that already had been presented without US. Bees in Group Plus show more response to $C$ at the end of SOC phase (Trials 4 and 5). Significant differences are marked with an asterisk.

increase for Group Minus $\left(\chi^{2}=3.56, P<0.06, \mathrm{~ns}, \mathrm{df}=1\right)$. Hence, within five SOC trials there was no extinction.

In order to replicate this finding, we repeated Experiment 1 with different experimenters using different odors (nonanone, 2-octanol, limonene; data not shown). Again, both groups (Group Plus, $n=109$; Group Minus, $n=108$ ) differed significantly in Trial $4(H=5.40, P<0.05, \mathrm{df}=1)$ and in Trial 5 $(H=5.94, P<0.05, \mathrm{df}=1$; data not shown $)$.

Because we obtained very similar results in two experiments, including the missing of extinction, we conclude that olfactory SOC in honeybees occurs when a new odor is forward-paired with an odor that had been conditioned earlier.

\section{Experiment 2: Does the order of pairing of the two odors presented during the SOC phase influence performance?}

The odors used as A and C were citral, geraniol, or 2-hexanal, and odor B was always nonanone. The three odors were equally balanced and used in all possible combinations to exclude odorspecific effects. The first column of Figure 2 shows that the acquisition curves for the differential conditioning protocol in Phase 1 are quite similar for both groups ( $n=70$ each). Bees from both groups showed $\sim 80 \%$ PER toward A and $<10 \%$ PER toward B after five trials. The panels in the middle column of Figure 2 show responses to the new odor $\mathrm{C}$ for forward-pairing with $\mathrm{A}(\mathrm{C}-\mathrm{A})$ in Group Forward (upper part) and for backward-pairing with A (A-C) in Group Backward (lower part). Comparisons withingroups by means of McNemar tests showed significantly increasing response $(15 \%-40 \%)$ from Trial 1 to Trial 5 for forwardpairing with $\mathrm{A}\left(\chi^{2}=10.26, P<0.001, \mathrm{df}=1\right)$ and significantly decreasing response $(55 \%-20 \%)$ from Trial 1 to Trial 5 for backward-pairing with A $\left(\chi^{2}=12.71, P<0.001, \mathrm{df}=1\right)$.

Between-group comparisons (Kruskal-Wallis ANOVA) revealed significant differences in Trial $1(H=26.04, P<0.001$, $\mathrm{df}=1)$ and Trial $5(H=9.47, P<0.01, \mathrm{df}=1)$. Thus, bees in Group Forward and Group Backward started into SOC from a different response level, and they also ended up at different levels.

In both groups, the \% PER to the stimuli was recorded in three parts: first stimulus alone, overlap, and then second stimu- lus alone. Therefore we were able to take a look at extinction of odor $\mathrm{A}$ in both groups (see Fig. 2, third column). Responding to A did not decrease from Trial 1 to Trial 5, neither for Group Forward $\left(\chi^{2}=0.00, \mathrm{~ns}, \mathrm{df}=1\right)$ nor for Group Backward $\left(\chi^{2}=0.66, \mathrm{~ns}, \mathrm{df}=1\right)$. Hence, within five SOC trials there was no extinction of $\mathrm{A}$.

The order of pairing of stimuli during the SOC phase had an influence on the performance during SOC. Forwardpairing (C-A) led to an increase in response to C. Backward-pairing (A-C) seemed to have had an inhibitory effect in response to $\mathrm{C}$ during SOC, although in both groups bees remained motivated by showing response to $\mathrm{A}$.

\section{Experiment 3: Backward SOC with} forward and backward pairings

The odors used as A and C were citral, geraniol, or 2-hexanal. The three odors were equally balanced and used in all possible combinations to exclude odorspecific effects. For all bees, the percent PER to the stimuli was recorded in three parts: first stimulus alone, overlap, and second stimulus alone. Since response during the overlap could be influenced by both stimuli and therefore is ambiguous, response during overlap is not shown in the figure.

The first column of Figure 3 shows response to odor $\mathrm{C}$ for forward-pairing with odor A (C-A) in Group Forward (upper part, $n=71$ ) and for backward-pairing with A (A-C) in Group Backward (lower part, $n=60$ ). The panels in the middle column show the acquisition curves for the differential conditioning protocol in Phase 2. The third column of Figure 3 shows response to stimulus $C$ that was never directly paired with the US.

During the initial pairing phase (Phase 1), we found no significant decrease with respect to response to $C$ in Trial 1 and Trial 5 , neither for Group Forward $\left(\chi^{2}=0.80, \mathrm{~ns}, \mathrm{df}=1\right)$ nor for Group Backward $\left(\chi^{2}=2.56, \mathrm{~ns}, \mathrm{df}=1\right)$. Responding to $\mathrm{C}$ differed in the last trial of the initial pairing phase between Group Forward and Group Backward $\left(\chi^{2}=6.96, P<0.01, \mathrm{df}=1\right)$.

In the differential conditioning phase (Phase 2) both groups showed an increase in response to A comparing Trial 1 and 5 (Group Forward: $\chi^{2}=40.20, P<0.001$, $\mathrm{df}=1$; Group Backward: $\left.\chi^{2}=27.38, P<0.001, \mathrm{df}=1\right)$. There was no difference between groups with respect to response to $\mathrm{A}$ in Trial $5\left(\chi^{2}=0.92, \mathrm{~ns}\right.$, $\mathrm{df}=1)$.

Comparisons within groups by means of Wilcoxon matched-pairs tests showed significantly increasing response to $\mathrm{C}$ from the last trial of Phase 1 to the first trial of the test phase for Group Forward with forward-pairing of C and A (C-A) in Phase 1 before differential conditioning of A in Phase $2(Z=3.62$, $P<0.001)$. For Group Backward, with backward-pairing of $C$ and A (A-C) in Phase 1 before differential conditioning of A in Phase 2 , the corresponding difference was not significant $(Z=0.30$, ns, $\mathrm{df}=1)$.

The association between $\mathrm{A}$ and $\mathrm{C}$ should be strongest in animals that successfully mastered differential conditioning in Phase 2 because only these animals learned that odor $\mathrm{A}$ is the only odor rewarded by a US during the whole experiment. Comparison of only those animals showed a significant difference between groups in the first test to $C$ in the test phase $\left(\chi^{2}=3.98\right.$, $P<0.05, \mathrm{df}=1)$. 


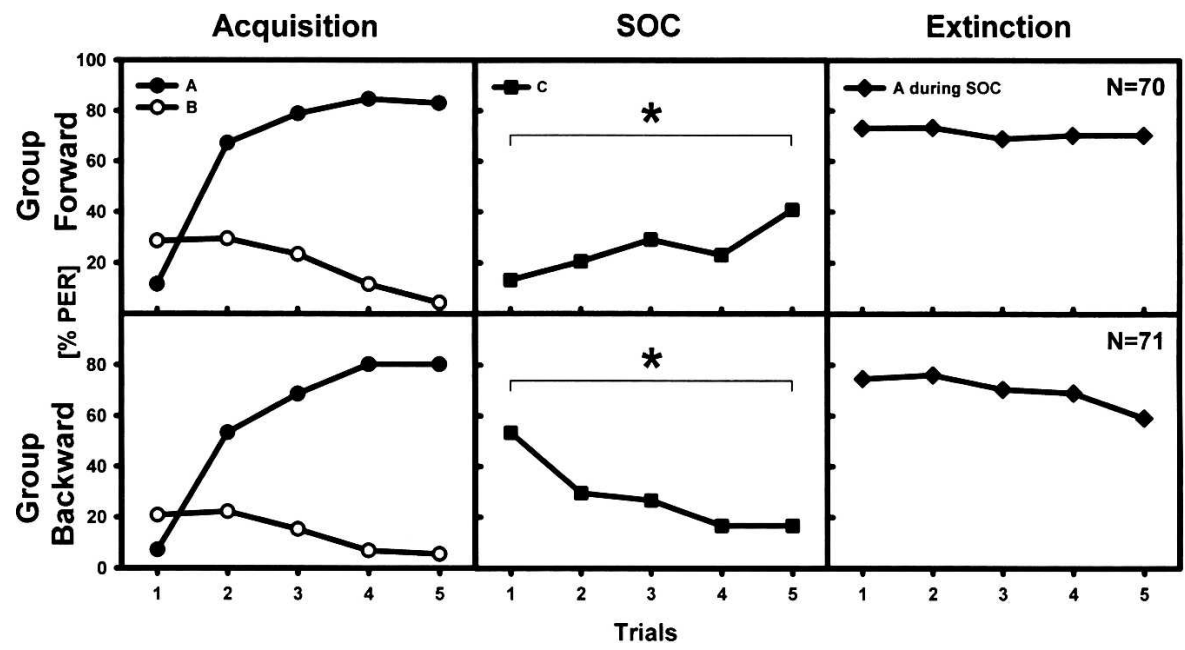

Figure 2. Contiguity and second-order conditioning $(\mathrm{SOC})$ in honeybees. Acquisition of odors $A$ and B (left panel) in a differential conditioning design (A-US, B-noUS). During the SOC phase (middle panel), Group Forward receives a forward-pairing of new odor $C$ with an already-conditioned excitatory odor A, while Group Backward receives a backward-pairing of A and C. Bees of Group Forward show an increase in response to $C$ during the $\mathrm{SOC}$ phase while bees of Group Backward show a decrease (comparing Trial 1 and Trial 5). With respect to responses to C during SOC, Group Forward and Group Backward differ significantly in Trial 1 and Trial 5. At the end of the SOC phase, the group that received a forward-pairing shows more responses to $C$ than the backward-paired group. The right panel shows the level of extinction of responses to A during the SOC phase. The tendency of extinction visible in Group Backward is not statistically different. Significant differences are marked with an asterisk.

\section{Discussion}

The experiments by Takeda (1961) and Bitterman et al. (1983) had already shown evidence of forward SOC, but Takeda's experiment did not exclude the possibility of sensitization as the cause for SOC, and in the Bitterman experiment very few animals $(n=10)$ were tested and a control group with backward-pairing was missing. In the present series of experiments, a large number of animals were tested, the odors were balanced during training, which eliminated the possibility of odor-specific effects, and effects due to successive presentation of two odors were evaluated by including backward-paired controls. Furthermore, the impact of the sequence of the firstorder and second-order protocol was looked at.

Experiment 1 showed that forwardpairing of a new odor $\mathrm{C}$ with a previously reinforced odor A led to higher response to $\mathrm{C}$ than did forward-pairing of a new odor $\mathrm{C}$ with a previously unreinforced odor B. This finding alone, however, cannot be interpreted as evidence for excitatory SOC. First, it might be argued that the mere presence of an excitatory CS in the SOC phase may have maintained motivation, alertness, and so forth, accounting for maintenance of response to the second-order CS in Group Plus. Second, the backward procedure as a well-known inhibitory learning procedure might have led to inhibitory SOC in Group Minus. Hence, the interpretation of the observed difference between groups has to be postponed.

Experiment 2 therefore examined

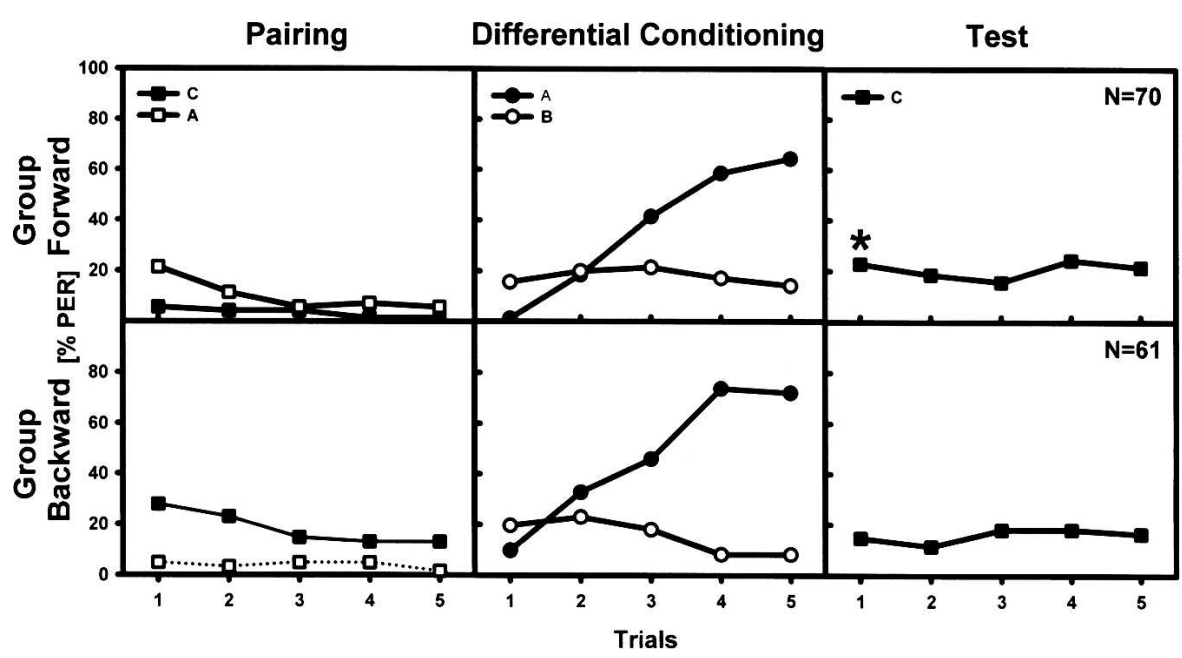

Figure 3. Backward second-order conditioning containing forward- and backward-pairing. The left panel shows response to $A$ and $C$ during the initial pairing phase. The second phase is differential conditioning (A-US, B-noUS; middle panel). In the last phase, responses to $C$ are tested (right panel). Initially, forward-pairing (Group Forward) shows a strong increase in responses to $C$ from the first to the last phase of the experiment. Backward-pairing (Group Backward) did not initially show any difference with respect to responses to $C$ between the first and the last phases. Classically conditioned odors prevent other odors from being learned if they are backward-paired before classical conditioning. Significant differences are marked with an asterisk. 
be seen in forward- and backward-pairings of odors (Experiment 2). The SOC was stronger in Experiment 2 compared to Experiment 1 because the first-order conditioning response was higher than in Experiment 1. This is supported by the fact that we found a positive correlation between first-order conditioning and SOC in Group Plus of Experiment $1(+0.43)$ and Group Forward of Experiment $2(+0.72)$. Odor A in Experiment 2 had a higher reinforcement value than odor A in Experiment 1 which was reflected in the subsequent SOC levels. Experiment 3 even showed successful backward SOC, at least for a stimulus that was followed by another stimulus (forward-pairing) before this other stimulus was paired with US.

In all differential conditioning experiments, training started with A-US and then B-noUS. Therefore, response toward B was always higher than toward A in the first trial. This is a generalization effect that is commonly observed in most of our differential conditioning experiments. Experiment 1 showed that SOC in olfactory PER conditioning in honeybees is a weak effect, because only about a quarter of the animals demonstrated that they had learned the new odor C. Nearly $75 \%$ of the bees do not change their learning pattern after SOC. SOC is much weaker than firstorder conditioning, where the percentage of animals showing learning is about three times higher. One reason for this might lie in the higher reinforcement value of the US sugar solution, since the primary reinforcer obviously provides a much higher US strength than an acquired US. Thus, the transmission of associative strength to $\mathrm{C}$ is weaker. Another potential cause for the reduced strength of an acquired US is extinction occurring due to repeated presentations of a previously reinforced odor without US (Bitterman et al. 1983; Menzel 1990; Hammer and Menzel 1998; Bonod et al. 2003; Stollhoff et al. 2005). In Group Plus of Experiment 1 and Group Forward of Experiment 2, however, there was no extinction during SOC. One would expect extinction of A in both groups, since A was repeatedly presented without US, and extinction has been frequently demonstrated in olfactory PER conditioning (see above). Since we did not include a control group that experienced an A-only stimulus in the second phase of conditioning, we cannot decide whether the lack of extinction in Experiment 2 can be related to the occurrence of a preceding C.

We chose a second group (Group Backward) to prove that the mere presence of a previously reinforced odor (A) is not sufficient to cause SOC, but instead a special sequence of two odors is necessary for SOC to occur. In Group Backward of Experiment 2 , a previously reinforced odor A preceded the new odor C (A-C). The bees learned to extend their proboscis at a high rate toward $\mathrm{A}$ in Phase 1 . In Phase 2 they continued to do so until the beginning of $\mathrm{C}$ (which overlapped with A). Remarkably, during Phase 2, the bees learned to retract the proboscis with the onset of new odor C (Fig. 2, lower middle panel). At Trial 5 the response to $\mathrm{C}$ in Group Backward was significantly lower than in Trial 1. Thus, backward-pairing of A with $\mathrm{C}$ led to a termination of the learned response at the onset of $\mathrm{C}$. While the same new odor $\mathrm{C}$ in Group Forward became a predictor for the occurrence of a learned odor, it developed an inhibitory property in Group Backward. Thus, SOC follows the rules of first-order conditioning: forward-pairing leads to excitatory conditioning, and backwardpairing to inhibitory conditioning. This finding emphasizes the special inhibitory meaning of backward-pairing in classical conditioning, as has been shown in other invertebrates as well (Tanimoto et al. 2004). Group Backward proved not only that the SOC effect cannot be explained as a generalization effect but also that the sequence of pairing (C-A vs. A-C) is of relevance in SOC.

Experiment 3 showed that in Phase 1 honeybees acquired associations between odors, even if no US occurred before or during acquisition. With respect to Group Forward, data showed that bees are able to acquire information about the relationship of different odors, even if these odors are presented without the presence of an US. A subsequent pairing with the US of one of the odors presented in Phase 1 makes the former learned association visible within a behavioral response. The fact that our data do not show an effect with respect to Group Backward does not mean that no association was learned between the two odors in Phase 1. It may only show that we did not choose the right testing procedure in order to show such associations. A summation test might possibly have been the proper test. Therefore one should have trained an odor $\mathrm{X}$ before the experiment only for the half of possible satiation (using only two learning trials). Afterward it would be possible to show excitatory or inhibitory influences of $\mathrm{C}$ to $\mathrm{X}$ within a mixture test.

In the last trial of Phase 1, response to $\mathrm{C}$ was higher in Group Backward than in Group Forward. This might be one reason that the increase in response from Phase 1 to Phase 3 failed to become significant in Group Backward and only became significant in Group Forward. The between-groups analysis of learners only, however, showed significantly higher response in Group Forward compared to Group Backward in Phase 3, although before learning in Phase 2 had taken place, response to $\mathrm{C}$ had been higher in Group Backward. Hence, there is some evidence that the increase in response is not due to a nonassociative effect.

Experiment 3 is quite similar to a set of experiments that were related to sensory preconditioning (Müller et al. 2000). In Müller's work, odors from Phase 1 were presented in a purely simultaneous way and in a sequential way, presenting one odor after the other without an overlap. Both groups are different from our experimental design. Müller et al. found significant effects for the simultaneous group but not for the sequential one. The experimental design used in our study is right in the middle of the groups used by Müller et al.: Odors were presented in a sequential way but with an overlap and therefore with a simultaneous part of odor presentations. Thus odor stimulation might just have been "simultaneously enough" to lead to learning of an association between the two odors. In general, backward SOC, as used in our study, might offer the opportunity to uncover earlier hidden learning, as in Phase 1 of Experiment 3. Pairing of a stimulus with an US provides the "energy" or "biological strength" that is necessary to map the already learned but so far hidden association between this stimulus and another one onto response.

The neural correlate of the reinforcing function in odor conditioning in bees, the neuron VUMmx1 (Hammer 1993, 1997; Menzel and Giurfa 2001) was found to require responses to the reinforced odor during acquisition, a property this neuron shares with dopamine neurons of the ventral tegmentum in the primate brain stem (Schultz 2006). Therefore, activation of the VUMmx1 neuron by the reinforced odor provides an internal reward signal under the control of this odor. Pairing a new odor with a previously reinforced odor in the SOC phase will, in fact, lead to a pairing of the new odor with an internal reward signal. Similarly, replacing the reward signal with a local injection of the putative transmitter of the VUMmx1 neuron (octopamine) leads to learning of the odor paired with the injection of the transmitter (Hammer and Menzel 1998). It is, therefore, reasonable to assume that SOC in bees is based on an internal reward signal that got under control during the initial acquisition phase.

\section{Acknowledgments}

B.K. was supported by grants from the German Research Council (DFG to H.L. [LA 564/10-4] and R.M. [ME 365/23-4]). S.A.H. was supported by GRK 837 (Graduate School "Functional Insect Science"). 


\section{References}

Amiro, T.W. and Bitterman, M.E. 1980. Second-order appetitive conditioning in goldfish. J. Exp. Psychol. Anim. Behav. Process. 6: $41-48$.

Bitterman, M.E., Menzel, R., Fietz, A., and Schäfer, S. 1983. Classical conditioning of proboscis extension in honeybees (Apis mellifera). J. Comp. Psychol. 97: 107-119.

Bonod, I., Sandoz, J.C., Loublier, Y., and Pham-Delègue, M. 2003. Learning and discrimination of honey odours by the honey bee. Apidologie (Celle) 34: 147-159.

Brembs, B. and Heisenberg, M. 2001. Conditioning with compound stimuli in Drosophila melanogaster in the flight simulator. J. Exp. Biol. 204: $2849-2859$.

Findley, J.D., Schuster, C.R., and Zimmerman, J. 1966. Second-order avoidance behavior in monkeys. J. Exp. Anal. Behav. 9: 703-708.

Galizia, C.G., Joerges, J., Kuttner, A., Faber, T., and Menzel, R. 1997. A semi-in-vivo preparation for optical recording of the insect brain. $J$. Neurosci. Methods 76: 61-69.

Hall, D. and Suboski, M.D. 1995. Sensory preconditioning and second-order conditioning of alarm reactions in zebra danio fish (Brachydanio rerio). J. Comp. Psychol. 109: 76-84.

Hammer, M. 1993. An identified neuron mediates the unconditioned stimulus in associative olfactory learning in honeybees. Nature 366: 59-63.

Hammer, M. 1997. The neural basis of associative reward learning in honeybees. Trends Neurosci. 20: 245-252.

Hammer, M. and Menzel, R. 1998. Multiple sites of associative odor learning as revealed by local brain microinjections of octopamine in honeybees. Learn. Mem. 5: 146-156.

Hawkins, R.D., Greene, W., and Kandel, E.D. 1998. Classical conditioning, differential conditioning, and second-order conditioning of the Aplysia gill-withdrawal reflex in a simplified mantle organ preparation. Behav. Neurosci. 112: 636-645.

Holland, P.C. and Rescorla, R.A. 1975. Second-order conditioning with food unconditioned stimulus. J. Comp. Physiol. Psychol. 88: 459-467.

Kamil, A.C. 1969. Some parameters of the second-order conditioning of fear in rats. J. Comp. Physiol. Psychol. 67: 364-369.
Komischke, B., Giurfa, M., Lachnit, M., and Malun, D. 2002. Successive olfactory reversal learning in honeybees. Learn. Mem. 9: 122-129.

Kuwabara, M. 1957. Bildung des bedingten reflexes von Pavlovs typus bei der honigbiene, Apis mellifica. J. Fac. Sci. Hokkaido Univ. Ser. VI Zool. 13: 458-464.

Menzel, R. 1990. Learning, memory, and "cognition" in honey bees. In Neurobiology of comparative cognition (eds. R.P. Kesner and D.S. Olton), pp. 237-292. Erlbaum, Inc., Hillsdale, NJ.

Menzel, R. and Giurfa, M. 2001. Cognitive architecture of a mini-brain: The honeybee. Trends Cogn. Sci. 5: 62-71.

Müller, D., Gerber, B., Hellstern, F., Hammer, M., and Menzel, R. 2000. Sensory preconditioning in honeybees. J. Exp. Biol. 203: 1351-1364.

Pavlov, I.P. 1927. Lectures on conditioned reflexes, pp. 103-114. International Publishers, New York.

Rescorla, R.A. 1979. Aspects of the reinforcer learned in second-order Pavlovian conditioning. J. Exp. Psychol. Anim. Behav. Process. 5: 79-95.

Sahley, C. 1981. An analysis of associative learning in a terrestrial mollusc. I. Higher-order conditioning, blocking and a transient US-pre-exposure effect. J. Comp. Physiol. 144: 1-8.

Schultz, W. 2006. Behavioral theories and the neurophysiology of reward. Annu. Rev. Psychol. 57: 87-115.

Stanhope, K.J. 1992. The representation of the reinforcer and the force of the pigeon's keypeck in first- and second-order conditioning. Q. J. Exp. Psychol. 44B: 137-158.

Stollhoff, N., Menzel, R., and Eisenhardt, D. 2005. Spontaneous recovery from extinction depends on the reconsolidation of the acquisition memory in an appetitive learning paradigm in the honeybee (Apis mellifera). J. Neurosci. 25: 4485-4492.

Takeda, K. 1961. Classical conditioned response in the honeybee. J. Insect Physiol. 6: 168-179.

Tanimoto, H., Heisenberg, M., and Gerber, B. 2004. Experimental psychology: Event timing turns punishment to reward. Nature 430: 983 .

Received November 14, 2006; accepted in revised form August 1, 2007. 


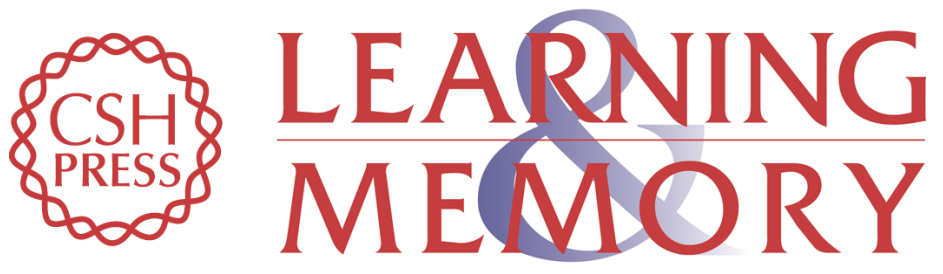

\section{Forward and backward second-order Pavlovian conditioning in honeybees}

Syed Abid Hussaini, Bernhard Komischke, Randolf Menzel, et al.

Learn. Mem. 2007, 14:

Access the most recent version at doi:10.1101//m.471307

References This article cites 24 articles, 4 of which can be accessed free at:

http://learnmem.cshlp.org/content/14/10/678.full.html\#ref-list-1

License

Email Alerting Receive free email alerts when new articles cite this article - sign up in the box at the Service top right corner of the article or click here. 